Methods applied: Routine lab, biomarkers (ELISA, HPLC, mass spectrometry, etc.), broad spectrum of obesity-related biomarkers with focus on adipokines, genetics (DNA, RNA, microRNAs, mitochondrial haplo- types), oxidative/nitrosative stress markers, carotids sonography, intima-media thickness, complex anthropometry including adipose tissue topography (lipometry).

\title{
Childhood Obesity: a Belgian Update
} The Belgian situation: a public health challenge

\author{
Michèle Guillaume
}

Head of the Department Nutrition, Environment and Health at the School of Public Health of the Faculty of Medicine of the University of Liege

Introduction: Childhood overweight and obesity remain a growing epidemic worldwide. During the past decades, the prevalence of overweight or obesity in children has doubled or tripled in several countries (Wang and Lobstein 2006). In Europe, the overall prevalence followed the same trend but with marked differences between countries (Lobstein and Frelut 2003). Here we present an update of the Belgian situation with respect to the international figures, as well as a review of environmental risk factors. What are the prevalence figures in Belgium?

Available data is difficult for comparisons because the age of the population studied and the definition used are different. At a national level, data based on self-reported height and weight were obtained from the Health Behaviour in School-aged Children study (HBSC). Results from the 2005-2006 HBSC study (WHO Regional Office for Europe 2008) for the French Community indicate that the prevalence of overweight or obesity, according to the International Obesity Task Force (IOTF) definition, amount $10 \%$ in both sexes at the age of 11 years, but increase to $10 \%$ and $14 \%$ at 13 years and to $12 \%$ and $15 \%$ at 15 years in girls and boys, respectively. Results were slightly higher than in previous surveys, although differences were not significant. In the Flemish Community, the prevalence of overweight or obesity was smaller than in the French Community, being $8 \%$ in both sexes at 11 years, 10\% in both sexes at 13 years and $8 \%$ and $11 \%$ for girls and boys, respectively, at 15 years of age. At a regional level, data are available from several studies in which height and weight were measured. In the province of Liège, a retrospective study was conducted based on school health records of 1403 children in 2005-2006. The mean age was 7 years (range: $2 \cdot 3-14 \cdot 6$ years). Using the IOTF definition, the prevalence of overweight or obesity was $16 \cdot 6 \%$ in girls and $15.9 \%$ in boys, and the prevalence of obesity was $4 \cdot 0 \%$ and $4 \cdot 4 \%$, respectively. In comparison with a previous study conducted in 1985, the prevalence of overweight albeit doubled in two decades (Guillaume and Counet 2009). In the province of Hainaut, a study conducted in 2003 revealed that the prevalence of overweight or obesity (according to the IOTF definition) was $25.9 \%$ in girls and $23.3 \%$ in boys at the age of 11 years (Observatoire de la Santé du Hainaut 2006). Finally, in the province of Luxembourg, a study conducted in 2004 (using the IOTF definition) showed that the prevalence of overweight or obesity was $25.6 \%$ in girls and $24.7 \%$ in boys at the age of 11 years (Counet et al. 2006). International comparison: the 2005-2006 HBSC study, which involved forty-one countries and regions, showed that the prevalence of overweight and obesity in Belgium ranked in the middle of the other countries, for both girls and boys (from 6\%, as in Netherlands, Lithuania and Romania, to $29 \%$ or $31 \%$, in the USA and Malta). Abdominal obesity is a real threat to health because it is a recognized risk factor for type 2 diabetes and CVD. Although it can already be detected in the late childhood and adolescence, to date, only a few studies have been performed among youngsters. In Belgium, a study conducted in 2008-2009, including 784 children of a mean age 11.5 ( $\mathrm{SD} 0.5$ ) years in the province of Liège, indicated that the prevalence of abdominal obesity varied between $15.4 \%$ according to the US percentile curves and $48.4 \%$ with the percentile curves of Great Britain. The prevalence was significantly higher in girls than in boys. According to these data, abdominal obesity in adolescents represents a significant public health problem in Liège, although the development of Belgian-specific reference curves would be required.

Risk factors: Several risk factors, such as genetic variability, are not modifiable but others, such as lifestyle and environmental risk factors, can be changed with prevention programs. However, these risk factors must be considered in a global context, both family-related and socio-economic.

Conclusions: Childhood overweight and obesity are a public health challenge in Belgium as they are worldwide. They require the involvement of all the actors of the society: politicians, health professionals, educational institutions, families, etc. The management should be multidisciplinary, centred on the child or the adolescent and their families. Since 2009, a research program called 
EDU-DORA2 (Education thérapeutique et préventive face au Diabète et à l'Obésité à Risque chez l'Adulte et l'Adolescent) is being conducted in Belgium, Luxemburg and Lorraine to investigate potential working tracks for a multifactorial and multidisciplinary approach in the management of childhood obesity (Scheen et al. 2010).

doi:10.1017/S1368980012001632

\title{
Drug therapy: actuality and perspectives
}

\author{
Daniel Brasseur
}

Head of the Medical Assessors, at the General Directorate Medicine of the Federal Ministry of Public Health of Belgium

The main role of regulators is to authorize the access to safe and efficacious medicinal products. The risk-benefit of a candidate drug should be positive for a specific disease and a target population. But is overweight or obesity as such a disease? Who should potentially be treated and be prescribed pharmacological agents? When so, what is considered to be a clinically relevant drug effect? Some answers are given for adults in 'Guideline on Clinical Evaluation of Medicinal Products used in Weight Control' by the Committee for Human Medicinal Products of the European Medicines Agency and for children in the 'Paediatric Addendum Guideline on Clinical Evaluation of Medicinal Products used in Weight Control'.

The simple conclusion of this guidance could well read: 'There is some room for drug therapy in childhood obesity, namely in severe cases and for limited duration, provided that the medicinal product is harmless and its effect sustained'. If indirect evidence demonstrates the risks linked to overweight, the impact of a drastic weight control on obesityrelated complications needs to be prospectively demonstrated in clinical trial conditions to define who will really benefit from a pharmacological treatment. Ideally the clinical relevance of a treatment allowing to lose weight (or to maintain an 'ideal' weight) should translate in less complications later in life, an increased life expectancy and a better quality of life also based on improved self-esteem. In practice such a composite endpoint is not achievable in pharmaceutical trials in an acceptable time frame. More realistically, the surrogate marker is weight (loss) accepted as a primary endpoint in most clinical trials (CT). The way to conduct CT is currently driven by the experience gained from past investigations in the field. Weight losses are often transient since partial failure or full relapses are frequent. Patients should be obese as defined, and have a documented history of failing to lose weight by means of lifestyle modifications, before enrolment into the pharmacological phase of a study. Further confounding factors related or not to the drug studied may affect the outcome of trials. Therefore regulators are de facto suspicious and require a robust methodology to circumvent bias as much as possible. The lessons learnt from the past have also contributed to carefully monitor the safety of weight losing agents. The guidance recommends collecting information during the whole trial period. Particular attention should be given to adverse events signalled from adult CT. This data set should notably encompass the adverse events related to lipid profile, liver function, cardiovascular system function and rebound phenomenon.

For centrally acting anorectic agents it is recommended that special attention and monitoring is afforded to neuropsychiatric events such as depression, sleep pattern and nightmares, assessment of self-esteem, aggression or suicidality. In growing children height velocity should also be monitored and pubertal development assessed by determining Tanner stage at baseline and endpoint. Today the tools for a quicker development of pharmacological agents are available (EC Paediatric Regulation 1901/2006) and can be used for future candidate drugs.

\section{'Over' or 'bad'-nutrition?}

\section{Marie Josee Mozin}

Founder member and Honorary President of the European Club of Paediatric Dieticians

Food intake and behaviour are considered the most important etiological factor of obesity in childhood and adolescence. The efficacy of dietary treatment depends on the regard for different stages designed to help the child and his family to modify on the long term their dietary habits, behaviour and lifestyle. 